\title{
HAND GESTURE DETECTION USING ARDUINO AND PYTHON FOR SCREEN CONTROL
}

\author{
Sarita K. Gavale \\ Department of Computer Engineering \\ R C Patel institute of technology, Shirpur, MH, India
}

\author{
Yogesh S. Jadhav \\ Department of mechanical \\ S S V P S BSD College of Engineering, Dhule
}

\begin{abstract}
Screen Controlled of the device is a one which can be dignified by physical programmed of human hand which is called as simple gesture. The user just necessities to apparel a gesture device which embraces a sensor. The sensor will record the movement of hand in a specific direction which will result in the movement of the robot in the respective direction. The robot and the Gesture device are connected wirelessly via radio waves . Gesture commands freely trainable by the user can be used for supervisory external devices with handheld wireless sensor unit, The persistence gesture acknowledgment in Computers has always been the minimization of the distance between the physical world and the digital world. The way humans interrelate among themselves could be employed in communication with the digital world by understanding gestures control via mathematical algorithm. Numerous ways and algorithms have been suggested and executed to achieve the goal of gesture recognition and its use in interconnecting with the digital world.
\end{abstract}

Gestures can be tracked using hand activities, accelerometers and more. The controller is interfaced with sensor to manipulate the path of the hand movement, The accelerometer depends upon the gestures of hand. Through accelerometer, a passage of data signal is received and it is processed with the help of Arduino microcontroller This paper content with the organized motion detection system and implementation of a gesture precise computer using Arduino, Uno ARDUINO with HCSR04, processor and a laptop loaded with Windows 10 operating system along with low cost hardware necessities. The system can be generally classified into two components: The Hardware part consisting of Arduino Microcontroller, the ultrasonic sensors HC-SR04 and a computer, preferably a laptop, and the software part consists of Arduino IDE and Python 3.8.2 IDLE with GUI module installed. The wireless message allows the user to interrelate with the advance technology

Keywords - Microcontroller, Python IDE, Arduino Uno, Sensor HCSR04, GUI library

\section{INTRODUCTION}

V..Sathananthavath proposed that There are mainly two existing types of gesture acknowledgement methods, i.e., vision-based and accelerometer and/before gyroscope based. Outstanding to the restrictions such as startling ambient optical noise, slower active response, and comparatively large data congregations /handling of vision-based method, our recognition system is realized based on MEMS acceleration sensors. Hence heavy computation burden would brought if gyroscopes was used for starting amount, there current system is founded on MEMS accelerometers

He after Concluding of the decision making a signal controlled robot that will be maneuvered by a hand gloved attached with the transmission circuit assembly [21]. The circuit assemblage will consist of accelerometer \& Arduino board beside with an Bee transmitter, which self-possessed function are an input device to the robot. The resolve of this venture is to regulator a robot with human hands to boost HMI. The robot is controlled based on gesture of hand, which develops simple for any person to tackle it. The basic operational principle for our robot is passage of the data signals of accelerometer interpretations to the arduino board fitted on the robot. The program compiled in that Sensor kit runs permitting to the accelerometer values, they makes the robot task consequently. While we have used threeaxis accelerometer, considering one axis will control the speed in accelerative or progressive direction and other two axes will control the revolving mechanism

Prajwal Ashwin Jawalekar suggested in the article of robot control by using hand gestures The key purpose of using hand gestures is that it suggestions a more illustration way of controlling the robot and with there feature robot can be handme-down as a wheelchair or as a spy robot or for that consciousness. As human hand gestures are ordinary, with the assistance of wireless communication, it is easier to interrelate with the robot in a more-friendly way. The robot's drive depends on the motions made by hand. The detached of this article is to form a wireless, hand gesture precise robot using an Sensor kit Uno ARDUINO, an accelerometer, and a RF transmitter as well as RF receiver established. The Sensor kit Uno ARDUINO microcontroller announces the similarity 
output values e.g., $\mathrm{x}$-axis and $\mathrm{y}$-axis values of the accelerometer and changes that analog value to individual digital value. The values are given a precise utility by this software. The digital values are managed by the Sensor kit Uno ARDUINO microcontroller and permitting to the tilt of the accelerometer sensor attached on the hand, it drives the instructions to the RF transmitter which sends the pointer to the receiver and there signals are handled by the receiver end which drives the motor to an actual direction in which we have set it to transport. The robot moves onward, deteriorating, right and left when we tilt our palm to forward, backward, right and left respectively and the robot halts when our palm is parallel to the pulverized.

He say in the conclusion hand-gesture-depend on interface for directing a car-robot. A operator can control a car-robot directly by using his or her hand motions. In the upcoming, I implement will directly use a mobile phone with an accelerometer to regulator a car-robot. I also want to add more hand gestures (such as the turn and slit) into the interface to control the car in a more natural and efficiently way.

Then from the Google open source we collected data regarding detection and gesture are the significant method for media between community beings just in the right way as keyboard and mouse play a role for interconnecting with computer. more of dominant techniques are presented for interface with computer and one of them is hand gesture recognizing system. In that system hand motion is import as an input which remove workability of mouse and keyboard shortcut keys. Hand motion and gesture is an smart and quick system. For recognizing user hand motion space ultrasonic sensors are used. By using hand gesticulation user can connected with computer easily and there is no necessary of any physical joining with user and system. There are same ways to confinement physical gesture there a computer would be capable for recognize. The signal can be caught using distance calculation, scam, or a information glove.

Gestures can also be captured via Bluetooth or infrared waves, Acoustic, Tangible, ophthalmic or motion technological means. The embedded had arrangements designed for specific control functions can be optimized to reduce the size and cost of the device and increase the reliability and performance. This project consists of mainly three components - Sensor kit Uno ARDUINO, Ultrasonic sensors, and a laptop. The ultrasonic sensors hooked to the Sensor kit are used to determine the gestures and the distance of the hand from the ultrasonic sensors. The code loaded in Sensor kit finds the individual keyword for the distance found and sends it to Windows OS. Python code that runs in the background recognizes the keywords and generates the corresponding virtual keystrokes for Windows. The hotkeys then control precise function of the application of intend to run, that is VLC Media Player.

Benjula Anbu Malar M B conclude that in research of Hand Gesture Control Robot ,the robotic actions via cord consistent with palm gesture. The robotic can pass approximately 300 meter. The expected efficiency is achieved with the above mentioned handheld device and in future we are aiming to replace the wired component with wireless technology. And it is expected to perform more efficiently such as increase in the distance travelled by the robot. This robots can be upgraded to detect human life styles earthquake and sweep by ways of enforcing the sensor therefore it can also be upgraded to bomb detecting robotic because it has robotic arm it may additionally elevate the bomb which is positioned at distant location. And these type of methodology can be used in rescue operation to view the sight without any complexity

\section{PROPOSED WORK}

Gesture detection is built on requiring finger position of hand as of the ultrasonic sensor. For dispensation the soft data, a micro-controller is compulsory; for that use Sensor kit UNO ARDUINO board. Via USB connection the microcontroller conveyances the organized and read distance value which is supported by the sensor of the associated. The data which is transfer by the ultrasonic sensor is working in the software in PC everyplace all the derivation are performed and the data is same with the predefined conditions (motion resolution). In this model ultrasonic sensors are place to detect hand position and are wired with the Sensor kit board .As considering ultrasonic sensor nonstop emanates sound and it gets reflected back from user's motion of hand. The distance with the sounds is transmission and recognition of reflect backward sound wave is unhurried from the micro-controller with board.

The motion detection of the hand is in definite by the ultrasonic sensor HCSR04. The range of this sensor is approximate to 1 to 13 feet. hand gesture controller for detecting the movement of the hand or we can say gesture of human activity. During test of sensor in propose work it also used the Sensor kit UNO ARDUINO kit it's important for processing the raw data by using microcontrollers. Sensor UNO ARDUINO kit is connected to the PC by using the USB port. microcontroller transfer the process and calculate distance value which is provided by sensor .and help programing language python 3.8.2 used py-auto GUI library that allows all data to control the input device of a computer such as a mouse and a keyboard. The sensor response to the data which is a process in software in the peripheral, which are the whole analysis is proficient and then data is coordinated with preplan situations. While doing all trial and testing procedure allows two ultrasonic sensors that detect the hand position whose connected to the Sensor kit. The ultrasonic sensor create a signal in the form of continuously tones and get evaluated back from the stoners hand. Doing this test used the Sensor kit IDE to execute module, IDE code as well as python code run to execute in the python program. The interaction between both modules ensued by inscription Sensor kit port number in python IDE 
This prototypical takes bellowing hardware apparatuses; there are same ultrasonic sensor and Sensor kit UNO ARDUINO board. In figure 5 shows the hand indication detection control of prototypical. System software consists Sensor kit IDE and python GUI. To run this prototypical, the python code would run proceeding python GUI initially. It match signals with preplanned situations and prints on python output shell as shown in figure: i).Collaboration between python and Sensor kit program occurs by writing Sensor kit port sequence with python program.

\begin{tabular}{|c|c|c|c|}
\hline $\begin{array}{c}\text { Hand } \\
\text { motion } \\
\text { detection }\end{array}$ & $\begin{array}{c}\text { Output } \\
\text { Created by } \\
\text { method }\end{array}$ & $\begin{array}{c}\text { Output } \\
\text { Created by } \\
\text { method }\end{array}$ & PDF \\
\hline $\begin{array}{c}\text { Hand arm } \\
\text { push in }\end{array}$ & Regressive & Regressive & Rise in image \\
\hline $\begin{array}{c}\text { Hand arm } \\
\text { pull out }\end{array}$ & Accelerative & Accelerative & $\begin{array}{c}\text { Rotate page } \\
\text { factual }\end{array}$ \\
\hline $\begin{array}{c}\text { Left hand } \\
\text { arm push in }\end{array}$ & Start & Page backward & $\begin{array}{c}\text { Rotate page } \\
\text { left }\end{array}$ \\
\hline $\begin{array}{c}\text { Left hand } \\
\text { arm pull out }\end{array}$ & Stop & Page forward & $\begin{array}{c}\text { Decrease out } \\
\text { image }\end{array}$ \\
\hline
\end{tabular}

Table 1 shows output command

Table 1 shows to the basic output of hand gesture by using sensor sensing to various action and position of human hand, right hand push in backward the output produced by system for video create zoom in of Portable document format

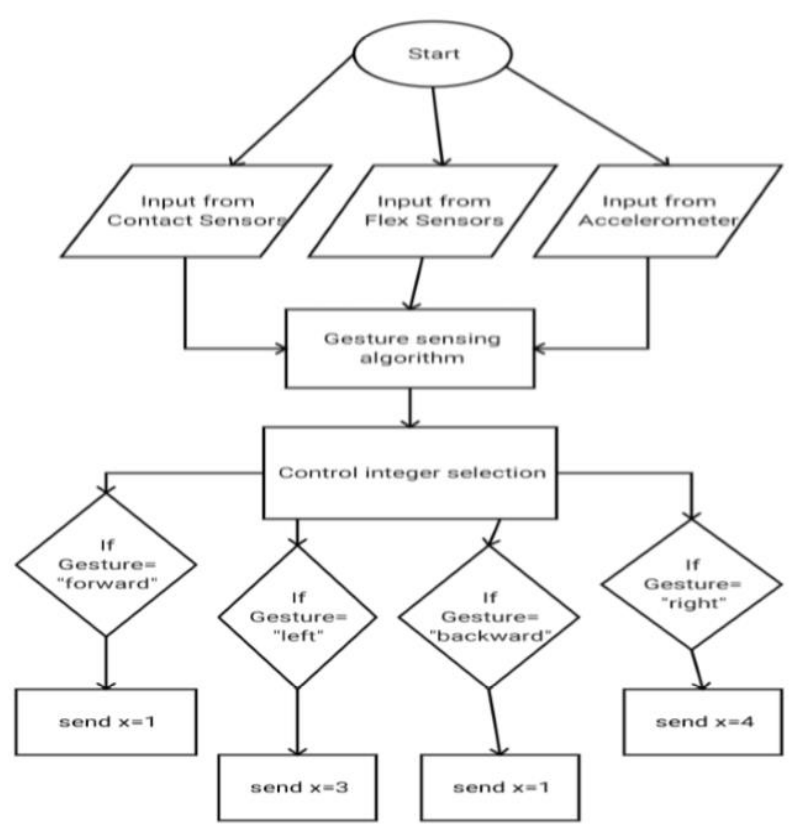

Fig .1 Flow chart for gesture sensing glove

The figure clarifies the system for operational of the motion controller. It obtains the control integer from the motion detecting glove and then uses that integer to decide which action is to be executed. Each integer value have allocated a specific action which is to be achieved by the wave.The motion is Superior Issue sensed using those input values and then a regulator integer is nominated for the gesture.

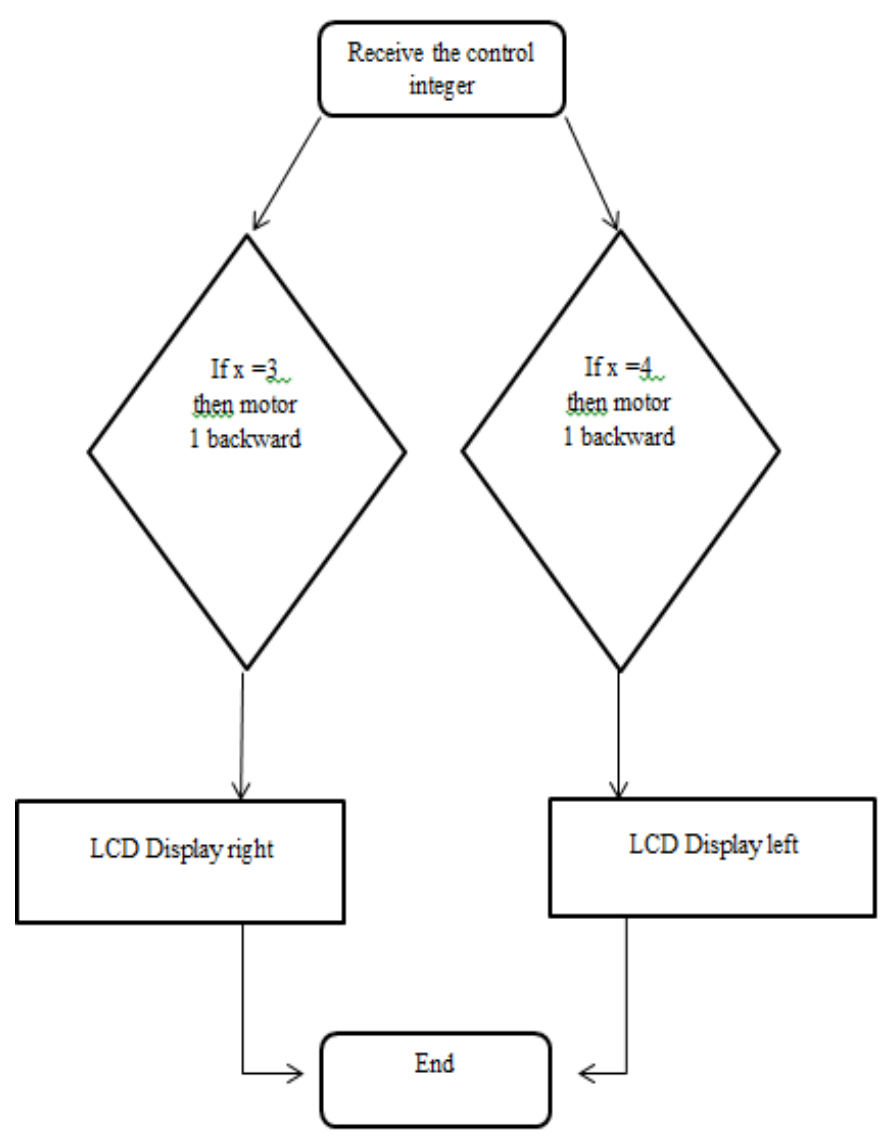

Fig. 2 Flow chart for program structure of gesture

Sensor kit Uno ARDUINO The Sensor kit Uno ARDUINO board have built on the ATmega328, Sensor kit UNO ARDUINO is an Open-Source Stage and soft to know for learners. A energetic character in Sensor kit is use by its common connecters which the CPU board wired with several addon modules called as shields., GPS, Ethernet, Liquid crystal diod, Motor controls or breadboard are delivered by shield. Sensor kit integrated development environment (IDE) is used to program Sensor kit boards in $\mathrm{C}$ and $\mathrm{C}++$ programming languages by a sequential connection.

Ultrasonic sensor is achieved to define distance to an object. It releases an ultrasound and this sound travels in the air. When it 
gets to an object it is moved back to the ultrasonic device . The device has four pins, they are, VCC, used to power afford sensor, Trigger, to conduct the US waves, and Echo means back sound, the yield pin .The US wave is come back to device use for detect sound waves from side to side eco pin as well as GND is connected with the ground pin of Sensor kit board. by the above figure 3.1, we observed that two ultrasonic sensors are wired with Sensor kit Uno ARDUINO board and the board is associated with Laptop through USB. The ultrasonic sensors have a receiver where the bringer discharges ultrasonic waves. The waves sensation a surface in front of the sensor and any reflected waves are take by the transmitter and based on the métier the distance of the object is calculated. This data is obtained by the Sensor kit and observed for different types of keywords inside the encryption permitting to the place that is to be transferred to the Python code running in the related of Windows system.

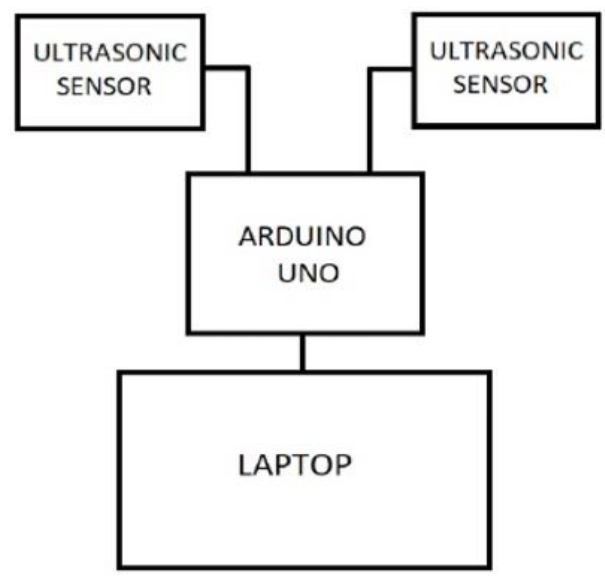

Fig. 3 block diagram for sensor connection

The ultrasonic sensors are the apparatuses that read the hand motion and he distance of the hand arm from the kit of all devices.

To read arm detection, will necessary to program an Sensor kit and project a Python code. As declared former that Windows does not recognize commands given from Sensor kit, so as we preferred Python to launch sequential collaboration and turn the data take into virtual keystrokes. virtual keystrokes switch the Hotkeys of VLC media player which in turn will govern playback of video or audio presence played. The Sensor kit IDE software appliance well for programming the Sensor kit but for Python we want to achieve a some steps after installing Python 3.8.2 as shown in Fig 4 The steps are Advancement pip function of Python 3.2.8 Install the PyAuto GUI module by pip function. Python code being executed allowing to inputs from Sensor kit . The program output of python 3.2.8 are shown in result.

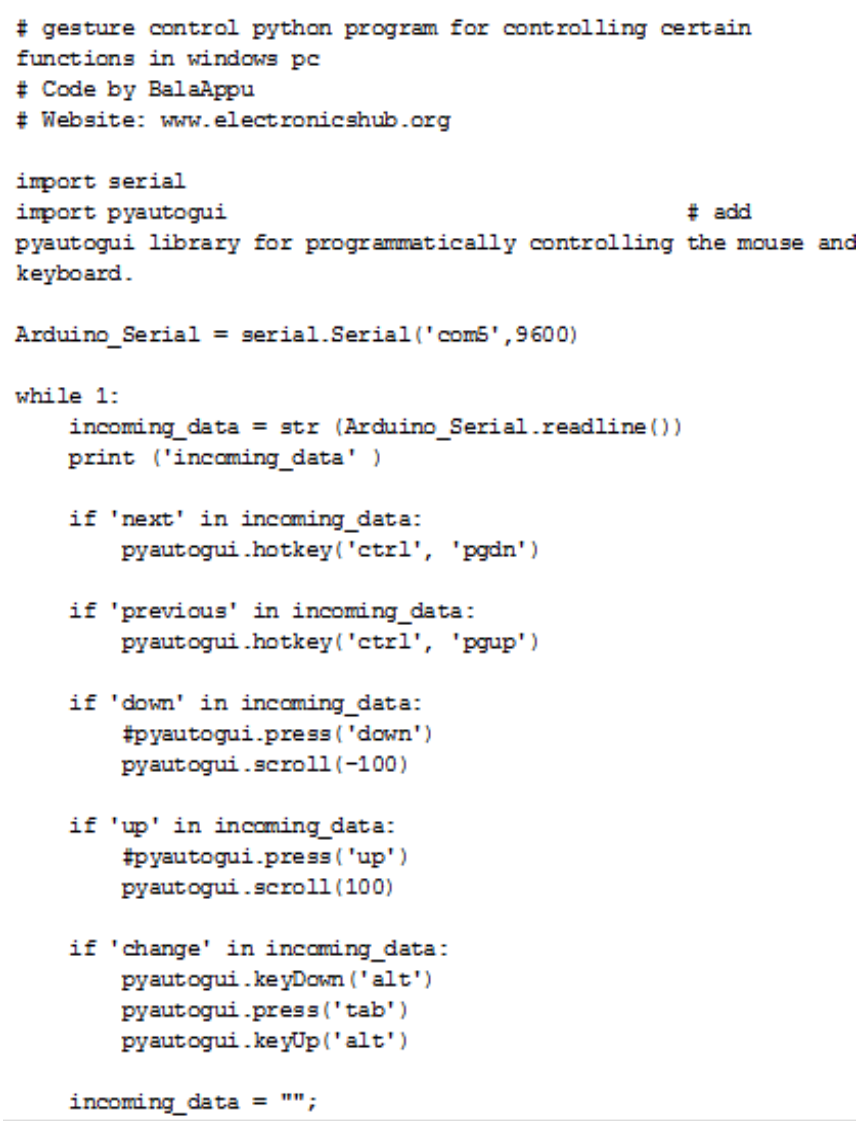

Fig. 4 Program for Gesture control by python code

\section{RESULT}

The detection rate of the system archived through this algorithm was 39It was noticed that the performance of the system improved as the data set given to neutral network(NN) for training was increased. For each type of gesture, 75 images were given to the system for training, The detection rate of system archived through this algorithm was 67 It was observed that the performance of the system improved as the data set for training was improved, In this technique ultrasonic sensor is used to sense hand motion gesture or of position of arm and conferring to situation action is accomplish on computer. The clarification shown in artifact is implementable and very beneficial for the operator. The use-case described it demonstrates the option of conforming virtual sensors inside a work cell. Whereas distance intention was preferred to find legal personnel by its location to work the robots, virtual devices are the impending for various utilization. The example of a few virtual model can remain overextended with color data, making it probable to detected QR-code stamps or same faces of the workers, or it is probable to use virtual sensors to record the velocity or acceleration of the human hand and position of arm, without actually loading hypothetically low capability equipment. In our paper we showed how we kept the digital print of a work cell up-to-date, manipulating data of presented 
sensor. We also practically organized how to citation evidence out of the digital twin, and how to contrivance digital sensor in a cyber-physical system. The obtained data have been effectively used in a motion and detection control scenario.

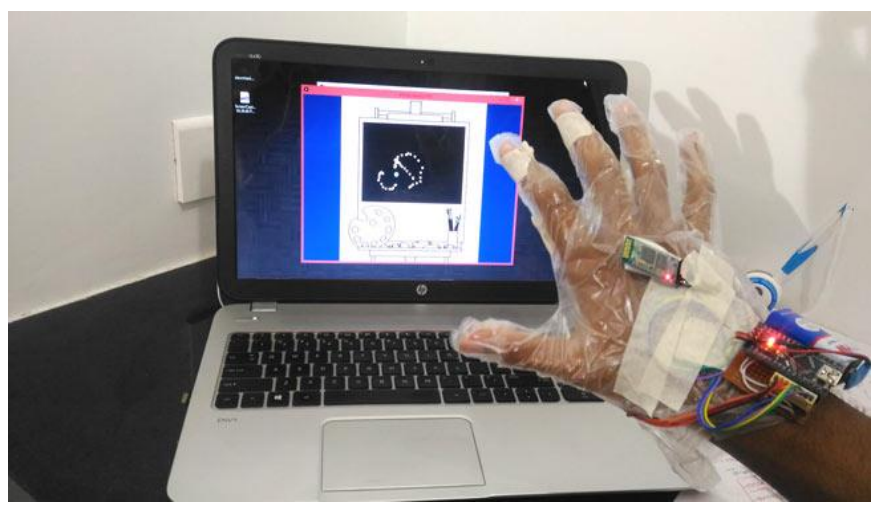

Fig. 5 Hand Gesture Controlled Laptop in action

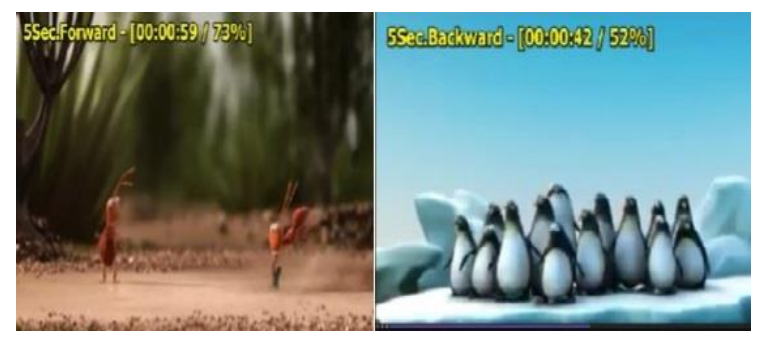

(a)

(b)

Fig. 6 (a) Show forward video (b) show backward video

\section{CONCLUSION}

In this paper we successfully trial the working of hand motion sensing system using sensors i.e. Ultrasonic sensors and finger contact sensors and using in it to Arduino kits in wireless mode using radio frequency. This can be applied in suffering situations for search and release or can be used for screen resolution detection and control to screen which again can be controlled using the glove. Some of the important features. This editorial presents one of the interpretation among various others, for operational a computer using hand motions. It is one of the easiest way of interface between human and computer .It is a budget effective model which is only based on Arduino UNO ARDUINO and python programming with wired ultrasonic sensor. The python IDE allows a continuous incorporation with it can be matching with most of the electronic devices, can be used for wide kind of applications from medical care to leisure, exact sensing of various gestures etc. The gesture sensing technique can also be used act of slide with control audio/ video clip making an collaborating way for deaf people to interact by giving the hand gestures, Regulatory the electronic devices in a house by different policy for monitoring and operating a smart phone.

\section{REFERENCE}

1) K. Sudheer,,et al November 2012, - International Journal of Research in Computer and Communication technologyl, IJRCCT, ISSN 2278-5841, Vol1, Issue 6,

2) C..Premangshu,M..PallabKanti; A. AsokeNath SubrataModak, 6, June 2016. "Gesture Controlled Robot using Arduino and Android”, IJARCSSE, Volume 6, Issue

3) T. Sandeep; M. Rajesh; B. Prakash, 2015. Vision based computer mouse control using hand gestures International Conference on Soft Computing Techniques and Implementations (ICSCTI)

4) Schlömer, T., Poppinga, B., Henze, N., Boll, S.: (2008)-Motion Recognition with a Wii Regulator.ll In: International Conference on Tangible and Embedded Interaction (TEI2008), Bonn Germany, Feburary 18-20, pp. 1114

5) Mäntyjärvi, J., Kela, J., Korpipää, P., Kallio, S.: (2004) -Enabling fast and effortless customization in accelerometer based gesture interaction. $\|$ In: Proceedings of the 3rd International Conference on Mobile and Ubiquitous Multimedia pp. 25-31.ACM Press, New York

6) Mantilla, V.-M.: (2001) —Discrete Hidden Markov Models with Application to Isolated User Dependent Hand Gesture Recognition.\| VTT publications

7) S. Zhou, Q. Shan, F. Fei, W. J. Li, C. P. Kwong, and C. K. Wu et al. Jan. 2009, - Gesture recognition for interactive controllers using MEMS motion sensors, $\|$ in Proc. IEEE Int. Conf. Nano/Micro Engineered and Molecular Systems, , pp. 935-940

8) S. Zhang, C. Yuan, and V. Zhang, Jul. 25th, 2008.-Handwritten character recognition using orientation quantization based on 3-D accelerometer,\| presented at the 5th Annu. Int. Conf. Ubiquitous Systems,

9) Hofmann, F.G., Heyer, P., Hommel, G.: (1998) -Velocity profile based recognition of dynamic gestures with discrete hidden markov models $\|$. In: Wachsmuth, I., Froehlich, M. (eds.) GW. LNCS, vol. 1371, pp. 81-95. Springer, Heidelberg

10) T. Yang, Y. Xu May 1994.,--Hidden Markov Model for Gesture Recognition\|, Robotics Institute, Carnegie Mellon Univ.,Pittsburgh, PA, CMU-RI-TR-94 10, 
11) O. Mirabella, M. Brischetto, G. Mastroeni May 2010-MEMS based gesture recognition\|, proc.HSI P.599 604 ,

12) Schlömer, T., Poppinga, B., Henze, N., Boll, S.: (2008)-Gesture Appreciation with a Wii Controller.l In: International Conference on Touchable and Embedded Interaction (TEI2008), Bonn Germany, , pp. 11-14

13) S. Greely; U. Tibor; K. Josef, 2016, Ost effective hand motion computer control interface ,IEEE International Conference on Cognitive Info communications.

14) C. J. Cohen; G. Beach; G.Foulk, (AIPR 2001). basic hand gesture control method for PC applications Chronicles 30th Applied Imagery Pattern Recognition Workshop

15) P. Loira; E. G.-Agulla, and J. L. Alba- Castro, 2014gestures to control infotainment equipment in cars ,. in Pros .IEEE Intell.Veh. Symp., , pp. 1.6.

16) Patil P.V., Shete M.B., Padalkar, T.M., : 04 , Apr2016. "Wireless Hand Movement Robot using Accelerometer, Volume: 03 Issue

17) B. Vivek, S. Girish Kumar, D. Sonu June 2015. "Motion Controlled Mobile Robotic Arm Using Accelerometer" in IJIRSET Vol. 4, Issue 6, 